\title{
Clinical applications of breast pathology: management of in situ breast carcinomas and sentinel node biopsy issues
}

\author{
Barbara L Smith \\ Massachusetts General Hospital, Gillette Center for Women’s Cancers, Boston, MA, USA
}

\begin{abstract}
The purpose of this article is to review the current clinical management of in situ breast carcinomas, including how specific aspects of a pathology report are used in clinical decision-making, and to discuss the current role of sentinel node biopsy in management of invasive breast carcinomas and ductal carcinoma in situ of the breast.

Modern Pathology (2010) 23, S33-S35; doi:10.1038/modpathol.2010.53
\end{abstract}

Keywords: sentinel node biopsy; breast cancer; ductal carcinoma in situ

Ductal carcinoma in situ of the breast (DCIS) is diagnosed with increasing frequency as a result of increasing use of screening mammography. The improved image quality obtained with digital mammography techniques has further enhanced identification of calcifications that indicate DCIS. The use of breast MRI has also increased diagnosis of DCIS and can identify areas of DCIS that are not associated with calcifications. Clinical management of DCIS is reviewed in the attached slides and Burstein et $a l^{1}$ have provided an excellent recent review.

\section{Sentinel node biopsy: reducing the morbidity of axillary staging}

The pathological status of axillary lymph nodes remains one of the most important prognostic factors in patients with breast cancer. Identification of metastatic tumor deposits in the axillary nodes indicates a poorer prognosis and often prompts a recommendation for more aggressive systemic and local therapies. Surgical staging of the axilla is a routine component of breast cancer treatment for the majority of the 200000 patients diagnosed with invasive breast cancer in the United States each year.

Correspondence: Dr BL Smith, MD, PhD, Gillette Center for Women's Cancers, Massachusetts General Hospital, Yawkey 9A, 55 Fruit Street, Boston, MA 02114, USA.

E-mail: blsmith1@partners.org

Received 03 February 2010; accepted 4 February 2010
Unfortunately, axillary dissection is often the main source of morbidity in patients with earlystage breast cancer. Immediate problems include acute pain, the need for hospital stay, reduced range of motion, and the need for a drain in the surgical bed for a week or more. Long-term problems resulting from axillary dissection include permanent lymphedema in up to $35 \%$ of patients, numbness in $35 \%$, chronic pain in $10 \%$, and reduced range of motion in $5-10 \% \cdot{ }^{2-13}$ An assessment of patients' subjective symptoms of arm problems shows even higher rates of persistent arm symptoms, with $25-50 \%$ of patients reporting arm swelling, pain, numbness, and/or decreased mobility. ${ }^{10,14-16}$ Studies of quality of life in patients treated for breast cancer have shown prolonged decreases in quality of life for those with lymphedema and other chronic arm symptoms resulting from axillary dissection. ${ }^{7-9,17}$

At present, there are no effective therapies to reverse established lymphedema. ${ }^{2}$ As lymphedema after axillary dissection cannot be 'cured', or even reliably prevented, identification of alternatives to standard axillary dissection have been sought.

The technique of sentinel node biopsy was developed with the aim of reducing the morbidity of surgical staging of the axilla. Identification of the first or 'sentinel' nodes draining the affected breast using radioactive particles ${ }^{18}$ or blue dye ${ }^{19}$ has allowed selective and minimally traumatic excision of only the most informative axillary nodes. Morbidity rates are substantially lower with sentinel node biopsy compared with axillary dissection. ${ }^{6,17-23}$ Acutely, sentinel node biopsy is an 
outpatient procedure that does not require a drain, allows rapid return to full mobility, and allows return to work weeks sooner than after axillary dissection. Longer-term morbidity is also reduced with a low rate of lymphedema, low incidence of numbness, and low rates of chronic pain. ${ }^{24}$

Sentinel node biopsy has been shown to provide reliable pathological staging of the axilla, with false negative rates generally $<5 \%$ in experienced hands. ${ }^{6,12,21}$ Axillary recurrence rates have been shown to be extremely low after a negative sentinel node biopsy without axillary dissection. ${ }^{6,12}$ A negative sentinel node biopsy is now widely accepted as sufficient to establish a patient as node-negative, with no further axillary treatment required.

\section{The role of sentinel node biopsy in DCIS}

Some authors have recommended routine sentinel node biopsy for DCIS or ductal carcinoma in situ with microinvasion (DCISM), as $8-12 \%$ of these patients are found to have nodal metastases on SNB, and up to $20 \%$ of patients with DCIS on their initial diagnostic biopsy are upstaged to invasive disease at definitive surgery.

Historically, survival for patients with DCIS and DCISM treated by breast conservation or mastectomy is $97-100 \%$, with deaths presumed to be due to spread of unrecognized or small numbers of invasive tumor cells. In invasive breast cancer, the major role of SNB is to identify patients whose tumor cells have spread beyond the breast, indicating higher risk for systemic disease. It has therefore been suggested to perform in patients with high-risk DCIS, meaning high risk of unrecognized invasive cancer, including those with microinvasion.

Several studies have helped identify sub-groups of patients at higher risk for occult invasive disease. Yen et $a l^{25}$ demonstrated that age $\leq 55$ years, diagnosis by core-needle biopsy, mammographic DCIS spanning $\geq 4 \mathrm{~cm}$, and high-grade DCIS were independent predictors of invasive cancer on final pathology, whereas only palpable tumor was predictive of a positive sentinel node. Tan et al. ${ }^{26}$ found that comedonecrosis and diagnosis by core-needle biopsy were independent risk factors for invasion in patients undergoing mastectomy and SNB for DCIS, but found no risk factors predictive for sentinel node metastases.

Although these studies have helped determine which DCIS patients are appropriate candidates for $\mathrm{SNB}$, few have examined the long-term outcomes in patients with sentinel node metastases. Broekhuizen et $a l^{27}$ examined 71 patients with DCIS and 12 with DCIS with $<2 \mathrm{~mm}$ invasion who had an excision of $\geq 5$ nodes and found that $11 / 83$ had positive axillary nodes by IHC or H\&E staining. Of positive lymph nodes, eight contained isolated tumor cells (ITCs), one had micrometastases, and two had macrometas- tases. At a median follow-up of 102 months, all patients remained free of disease. Moore et al. ${ }^{28}$ found $43(9.1 \%)$ positive sentinel nodes in 470 patients with high-risk DCIS: 36 with ITCs, 4 with micrometastases, and 3 with macrometastases. Of 25 patients who underwent ALND, only 1 was found to have additional positive nodes. No local recurrences were observed, but one patient with ITCs developed distant metastases at 27 months. Intra et al examined 854 patients with pure DCIS (microinvasion excluded) who had undergone SNB and found 4 with ITCs, 7 with micrometastases and 5 with macrometastases. Of these, 11 patients underwent ALND with no additional positive nodes. At a median follow-up of 41 months, there were two locoregional recurrences and one distant recurrence in patients with positive SNBs. The size of nodal deposits in these patients was not reported.

Murphy et $a l^{29}$ reported a series of 322 patients with DCIS and DCISM and found positive SNBs in $9 \%$ of patients, with the majority being $\mathrm{pNo}(\mathrm{i}+)$. At a median follow-up of 47.9 months there were 13 $(4.0 \%)$ local recurrences and 1 distant recurrence, all but one in sentinel node-negative patients.

El-Tamer et $a l^{30}$ demonstrated that positive lymph nodes detected by IHC in patients with DCIS did not alter breast cancer recurrence or survival.

\section{Rational use of sentinel node biopsy in DCIS}

In the Protocol for the Examination of Specimens from Patients with DCIS of the Breast recently prepared by Lester et $a l^{31}$ for the Members of the Cancer Committee, College of American Pathologists, it is suggested that patients with DCIS may have lymph nodes sampled in the following situations:

- Extensive DCIS: Patients with extensive DCIS are more likely to have areas of invasion and it may be difficult or impractical to examine all involved areas of the breast microscopically. A lymph node with a macrometastasis would indicate an occult area of invasion.

- Pathological findings based on a previous needle biopsy or excision raising concern for invasion or microinvasion (invasion measuring $\leqslant 0.1 \mathrm{~cm}$ in size): If invasion has been documented, the checklist for invasive carcinoma of the breast should be used.

- Imaging findings (eg, an irregular mass) or clinical findings (eg, a large palpable mass) that increase the likelihood that stromal invasion is present.

- Planned mastectomy: The additional sampling of low lymph nodes or a sentinel lymph node does not result in increased morbidity. If the node or nodes are negative, and invasive cancer is found, another surgical procedure for node sampling can be avoided. 


\section{Disclosure/conflict of interest}

The author declares no conflict of interest.

\section{References}

1 Burstein HJ, Polyak K, Wong JS, et al. Ductal carcinoma in situ of the breast. N Eng J Med 2004; 350:1430-1441.

2 Erickson VS, Pearson ML, Ganz PA, et al. Arm edema in breast cancer patients. [Review] [115 refs]. J Natl Cancer Inst 2001;93:96-111 (UI: 11208879).

3 Kissin MW, Querci G, Rovere della, et al. Risk of lymphedema following the treatment of breast cancer. Br J Surg 1986;73:580-584.

4 Larson D, Weinstein M, Goldberg I, et al. Edema of the arm as a function of the extent of axillary surgery in patients with stage I-II carcinoma of the breast treated with primary radiotherapy. Int J Radiat Oncol Biol Phys 1986;12:1575-1582 (UI: 3759582).

5 Siegel BM, Mayzel KA, Love SM. Level I and II axillary dissection in the treatment of early stage breast cancer. Arch Surg 1990;125:1144-1147.

6 Blanchard DK, Donohue JH, Reynolds C, et al. Relapse and morbidity in patients undergoing sentinel lymph node biopsy alone or with axillary dissection for breast cancer. Arch Surg 2003;138:482-487; discussion 487488 (UI: 12742949).

7 Kwan W, Jackson J, Weir LM, et al. Chronic arm morbidity after curative breast cancer treatment: prevalence and impact on quality of life. J Clin Oncol 2002;20:4242-4248 (UI: 12377968).

8 Beaulac SM, McNair LA, Scott TE, et al. Lymphedema and quality of life in survivors of early-stage breast cancer. Arch Surg 2002;137:1253-1257 (UI: 12413312).

9 Engel J, Kerr J, Schlesinger-Raab A, et al. Axilla surgery severely affects quality of life: results of a 5-year prospective study in breast cancer patients. Breast Cancer Res Treat 2003;79:47-57 (UI: 12779081).

10 Voogd AC, Ververs JM, Vingerhoets AJ, et al. Lymphoedema and reduced shoulder function as indicators of quality of life after axillary lymph node dissection for invasive breast cancer. Br J Surg 2003;90:76-81 (UI: 12520579).

11 Petrek JA, Heelan MC. Incidence of breast carcinomarelated lymphedema [review]. Cancer 1998;83(Suppl American):2776-2781.

12 Giuliano AE, Haigh PI, Brennan MB, et al. Prospective observational study of sentinel lymphadenectomy without further axillary dissection in patients with sentinel node-negative breast cancer. J Clin Oncol 2000;18:2553-2559 (UI: 10893286).

13 Duff M, Hill ADK, McGreal G, et al. Prospective evaluation of the morbidity of axillary clearance for breast cancer. Br J Surg 2001;88:114-117.

14 Armer JM, Radina ME, Porock D, et al. Predicting breast cancer-related lymphedema using self-reported symptoms. Nurs Res 2003;52:370-379 (UI: 14639083).

15 Pain SJ, Vowler SL, Purushotham AD. Is physical function a more appropriate measure than volume excess in the assessment of breast cancer-related lymphoedema (BCRL)? Eur J Cancer 2003;39:21682172 (UI: 14522374).
16 Ivens D, Hoe AL, Podd CR, et al. Assessment of morbidity from complete axillary dissection. Br J Cancer 1992;66:136-138.

17 Jani AB, Basu A, Heimann R, et al. Sentinel lymph node versus axillary lymph node dissection for earlystage breast carcinoma: a comparison using a utilityadjusted number needed to treat analysis. Cancer 2003;97:359-366 (UI: 12518360).

18 Krag DN, Weaver DL, Alex JC, et al. Surgical resection and radiolocalization of the sentinel lymph node in breast cancer using a gamma probe. Surg Oncol 1993;2:335-340.

19 Giuliano AE, Kirgan MD, Guenther JM, et al. Lymphatic mapping and sentinel lymphadenectomy for breast cancer. Ann Surg 1994;220:391-401.

20 Albertini JJ, Lyman GH, Cox C, et al. Lymphatic mapping and sentinel node biopsy in the patient with breast cancer. JAMA 1996;276:1818-1822.

21 Veronesi U, Paganelli G, Galimberti V, et al. Sentinelnode biopsy to avoid axillary dissection in breast cancer with clinically negative lymph-nodes. Lancet 1997;349:1864-1867.

22 Giuliano AE, Dale PS, Turner RR, et al. Improved axillary staging of breast cancer with sentinel lymphadenectomy. Ann Surg 1995;222:394-401.

23 Golshan M, Martin WJ, Dowlatshahi K. Sentinel lymph node biopsy lowers the rate of lymphedema when compared with standard axillary lymph node dissection. Am Surg 2003;69:209-211 (UI: 12678476).

24 Temple LK, Baron R, Cody III HS, et al. Sensory morbidity after sentinel lymph node biopsy and axillary dissection: a prospective study of 233 women. Ann Surg Oncol 2002;9:654-662.

25 Yen TW, Hunt KK, Ross MI, et al. Predictors of invasive breast cancer in patients with an initial diagnosis of ductal carcinoma in situ: a guide to selective use of sentinel lymph node biopsy in management of ductal carcinoma in situ. J Am Coll Surg 2005;200:516-526.

26 Tan JC, McCready DR, Easson AM, et al. Role of sentinel lymph node biopsy in ductal carcinomain-situ treated by mastectomy. Ann Surg Oncol 2007;14:638-645.

27 Broekhuizen LN, Wijsman JH, Peterse JL, et al. The incidence and significance of micrometastases in lymph nodes of patients with ductal carcinoma in situ and T1a carcinoma of the breast. Eur J Surg Oncol 2006;32:502-506.

28 Moore KH, Sweeney KJ, Wilson ME, et al. Outcomes for women with ductal carcinoma-in-situ and a positive sentinel node: a multi-institutional audit. Ann Surg Oncol 2007;14:2911-2917.

29 Murphy CD, Jones J, Javid SH, et al. Do sentinel node micrometastases predict local recurrence risk in ductal carcinoma in situ and ductal carcinoma in situ with microinvasion? Am J Surg 2008;196:566-568.

30 El-Tamer M, Chun J, Gill M, et al. Incidence and clinical significance of lymph node metastasis detected by cytokeratin immunohistochemical staining in ductal carcinoma in situ. Ann Surg Oncol 2005;12:254-259.

31 Lester SC, Bose S, Chen Y-Y, , et al., for the Members of the Cancer Committee, College of American Pathologists. Protocol for the Examination of Specimens from Patients with Ductal Carcinoma In situ (DCIS) of the Breast. DCIS Breast 2008 Version 11 (May, 2008). 